\title{
Specificity of two monoclonal antibodies against a synthetic glycopeptide, an analogue to the hypo-galactosylated IgA1 hinge region
}

\author{
Yoshiyuki Hiki · Hideo Hori • Kouichiro Yamamoto • \\ Yoshihiro Yamamoto • Yukio Yuzawa • \\ Nobuya Kitaguchi • Kazuo Takahashi
}

Received: 14 April 2014 / Accepted: 26 June 2014/Published online: 19 July 2014

(C) The Author(s) 2014. This article is published with open access at Springerlink.com

\begin{abstract}
Increased levels of hypo-galactosylated immunoglobulin (Ig)A1 (HG-IgA1) in IgA nephropathy (IgAN) have been detected using a Helix aspersa agglutinin lectin enzyme-linked immunosorbent assay (ELISA). In this study, we developed monoclonal antibodies to evaluate the HG-IgA1 in IgA nephropathy, aiming to gain a more consistent and reproducible assay. As an analogue to the HG-IgA1 hinge region, a 19 mer synthetic peptide with five GalNAc (sHGP) residues at positions 4, 7, 9, 11 and 15 [VPST(GalNAc)PPT(GalNAc)PS(GalNAc)PS(GalNAc) TPPT (GalNAc)PSPS-NH2] was synthesized. Two monoclonal antibodies against sHGP (35A12 and 44H8) that reacted with human IgA were developed. Also, their reactivities to serum $\operatorname{IgA}$ from $\operatorname{IgAN}$ patients $(n=49)$, patients with other forms of kidney diseases (OKD, $n=48$ ), and healthy controls ( $\mathrm{HC}, n=41$ ) were evaluated using ELISA assays. The binding levels of the two monoclonal antibodies against serum IgA were significantly higher (all comparisons, $p<0.0001$, Steel-Dwass non-parametric test) in IgAN patients compared to $\mathrm{HC}$ and OKD patients. In each individual, there was a close correlation of $\operatorname{IgA}$ binding levels between $35 \mathrm{~A} 12$ and $44 \mathrm{H} 8\left(R^{2}=0.737\right)$. These
\end{abstract}

Y. Hiki and H. Hori contributed equally to this study.

Y. Hiki $(\bowtie) \cdot$ H. Hori · N. Kitaguchi

Department of Clinical Engineering, School of Health Sciences,

Fujita Health University, 1-98 Dengakugakubo, Kutsukake-cho,

Toyoake, Aichi 470-1192, Japan

e-mail: yoshi-cake@mtj.biglobe.ne.jp

K. Yamamoto $\cdot$ Y. Yamamoto $\cdot$ Y. Yuzawa $\cdot$ K. Takahashi Department of Nephrology, School of Medicine, Fujita Health University, 1-98 Dengakugakubo, Kutsukake-cho, Toyoake, Aichi 470-1192, Japan results indicate that the monoclonal antibodies recognize similar epitopes in HG IgA1, which is found predominantly in IgAN patients. The developed antibodies are proposed as a clinically useful tool for IgAN screening.

Keywords IgA nephropathy $\cdot \operatorname{IgA} 1$ hinge region . $\mathrm{N}$-acetylgalactosamine $\cdot$ Galactose $\cdot$ Monoclonal antibody

$\begin{array}{ll}\text { Abbreviations } & \\ \text { IgA } & \text { Immunoglobulin A } \\ \text { IgAN } & \text { IgA nephropathy } \\ \text { GalNAc } & N \text {-acetylgalactosamine } \\ \text { sHGP } & \text { Synthetic hinge glycopeptide } \\ \text { HC } & \text { Healthy controls } \\ \text { OKD } & \text { Other forms of kidney diseases } \\ \text { HAA } & \text { Helix aspersa agglutinin } \\ \text { HPLC } & \text { High performance liquid } \\ & \text { chromatography } \\ \text { MALDI-TOF-MS } & \text { Matrix-assisted laser desorption } \\ & \text { ionization time of flight mass } \\ & \text { spectrometry } \\ \text { KLH } & \text { Keyhole limpet hemocyanin } \\ \text { AUC } & \text { Area under the curve } \\ \text { ROC } & \text { Receiver operating characteristic }\end{array}$

Introduction

Immunoglobulin ( $\mathrm{Ig}) \mathrm{A}$ nephropathy (IgAN) diagnosis is currently confirmed using an invasive method of renal biopsy, as the presence of predominant mesangial $\operatorname{IgA}$ deposits is the gold standard for diagnosis. Several candidates of serum and urinary markers for identifying $\operatorname{Ig} \mathrm{A}$ nephropathy have been proposed, such as anti-IgA antibody [1, 2] and anti-IgA1 hinge peptide antibody [3, 4]. 
However, they exhibit insufficient specificity for clinical application to identify IgAN.

There is increasing evidence supporting the involvement of aberrantly glycosylated $\operatorname{IgA} 1$ in the pathogenesis of IgAN [5-12]. It has been suggested that the presence of truncated $O$-glycans with an exposed GalNAc residue is more common on the IgA1 of IgAN patients. Previously, $O$-glycans in the hinge region of IgA1 were evaluated with an enzyme-linked immunosorbent assay (ELISA) using the Helix aspersa agglutinin (HAA) lectin, which specifically binds to GalNAc residues [13, 14]. The results indicated that there is increased binding of HAA to serum IgA1 from IgAN patients. In a previous study, we produced a rabbit polyclonal antibody against a synthetic IgA1 hinge peptide containing five GalNAc residues as an analogue of galactose-deficient IgA1 hinge region. The polyclonal antibody was capable of binding to serum IgA1 but poorly identified IgAN [15]. In this study, therefore, we attempted to develop monoclonal antibodies that specifically recognize hypo-galactosylated $\operatorname{IgA} 1$, which is found predominantly in $\operatorname{IgAN}$ patients.

\section{Materials and methods}

\section{Patients and test sera}

The study was approved by the ethical committee (No.14053) of Fujita Health University in accordance with the Helsinki Declaration. Forty-nine patients with biopsy-proven IgAN were examined. An additional 48 patients with other kidney diseases (OKD) and 41 healthy subjects (HC) were enrolled in the study as disease and healthy controls, respectively. The number of OKD patients and their disease details are reported in Table 1. The HC subjects were selected from healthy individuals who were gender- and

Table 1 Itemization of patients with other kidney diseases (OKD)

\begin{tabular}{ll}
\hline Disease & $\begin{array}{l}\text { Number of } \\
\text { patients }\end{array}$ \\
\hline Lupus nephritis & 22 \\
Anti-neutrophil cytoplasmic autoantibody & 11 \\
$\quad$ associated nephritis & \\
Membranous nephropathy & 4 \\
Minimal change nephrotic syndrome & 2 \\
Focal segmental glomerulosclerosis & 3 \\
Nephrosclerosis & 2 \\
Amyloidosis & 2 \\
Cholesterol crystal embolism & 1 \\
Diabetic nephropathy & 1 \\
Total & 48 \\
\hline
\end{tabular}

age-matched to the IgAN patients. All sera samples were stored at $-80{ }^{\circ} \mathrm{C}$ until use.

\section{Antigen}

A 19 mer synthetic peptide with five GalNAc (sHGP) residues at positions 4, 7, 9, 11 and 15 [VPST(GalNAc) PPT(GalNAc)PS(GalNAc)PS(GalNAc)TPPT(GalNAc)PS PS-NH2] was purchased from the Peptide Institute, Inc. (Osaka, Japan). The GalNAc glycosylation sites were determined according to Mattu et al. [16], who found that GalNAc residues are frequently located at these sites in the human IgA1 hinge region. The purity and molecular weight were confirmed by high performance liquid chromatography (HPLC) and matrix-assisted laser desorption ionization time of flight mass spectrometry (MALDI-TOF-MS). For immunization, the sHGP was conjugated with keyhole limpet hemocyanin (KLH; Sigma Chemical Company, St. Louis, MO, USA).

Monoclonal antibodies

Monoclonal antibodies against sHGP-KLH were produced by Tomiyama Laboratory Co. Ltd. (Tokyo, Japan) using conventional procedures. Briefly, $100 \mu \mathrm{g}$ of sHGP-KLH was subcutaneously injected into a BALB/cA mouse (6week-old female, CLEA Japan, Inc. Shizuoka, Japan) three times, with 2 weeks between injections. Three days after the final immunization, spleen and lymph nodes were collected from the mouse under general anesthesia. The lymphocytes isolated from the immunized mouse were mixed with myeloma cells at a ratio of 5:1. Initially, the mixed cells were gently suspended in $1 \mathrm{ml}$ of $50 \%$ polyethylene glycol (PEG) solution (PEG 1500, Roche Diagnostic Corp., Indianapolis, USA) for cell fusion, and subsequently resuspended in RPMI-1640 (Gibco Life Technologies, Grand Island, NY, USA) containing $15 \%$ FBS (Biowest, Nuaille, France) and hypoxanthine aminopterin thymidine (HAT) supplement (Gibco Life Technologies). The cells were incubated in a 96-well culture plate for 1 week in a $\mathrm{CO}_{2}$ incubator for cell proliferation. Antibody screening was performed using ELISA to select culture supernatants, producing antibodies that reacted with sHGP and serum IgA, but not with synthetic hinge peptide without GalNAc residues (sHP). After cloning of the hybridoma cells, hybridoma implantation was performed and ascites were collected. As a result, six monoclonal antibodies [35A12

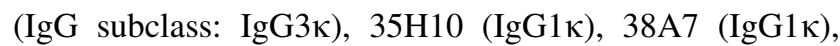

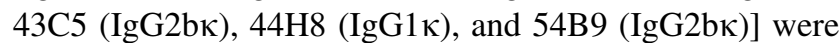
raised as candidates to identify serum hypogalactosylated IgA1. The IgG fractions were isolated from ascites using conventional ion-exchange chromatography. From the pilot study using a small number of IgAN and HC sera, we 
ultimately selected two monoclonal antibodies, 35A12 and $44 \mathrm{H} 8$, as convincing markers of IgAN.

We constructed dose-response curves for IgA binding to the monoclonal antibodies, 35A12 and 44H8, using serially diluted $(1 / 25-1 / 6,400)$ neuraminidase-treated human serum samples (Fig. 1a, b).

\section{ELISA assay}

Serum IgA binding levels to each monoclonal antibody were compared among the IgAN, OKD and HC groups using the following ELISA assay. A $50 \mu \mathrm{l}$ aliquot of $0.05 \mathrm{M}$ bicarbonate buffer ( $\mathrm{pH} 9.6$ ) containing $10 \mu \mathrm{g} / \mathrm{ml}$ of monoclonal antibody was added to the wells of a 96-well polystyrene microtiter plate (Corning Inc., Corning, NY, USA) and incubated overnight at $4{ }^{\circ} \mathrm{C}$. The plates were washed with $0.01 \mathrm{M}$ phosphate buffered saline (PBS)-0.05\% Tween-20 (pH 7.4, PBST) three times at each step. Coated plates were blocked with $1 \%$ bovine serum albumin (BSA; Sigma Chemical Company) in PBS for $1 \mathrm{~h}$ at room temperature (RT). The coated plates were stored at $4{ }^{\circ} \mathrm{C}$.

Removal of terminal neuraminic acid from the IgA1 glycans, leaving terminal GalNAc residues, was accomplished by incubating serum samples with five volumes of $0.01 \mathrm{M}$ acetate buffer ( $\mathrm{pH} \mathrm{5)}$ containing $1.5 \mathrm{mU} / \mathrm{ml}$ neuraminidase (from Vibrio cholerae, Roche Diagnostic Corp., Indianapolis, IN, USA) for $4 \mathrm{~h}$ at $37{ }^{\circ} \mathrm{C}$ in micro-tubes ( $1.5 \mathrm{ml}$; Crystalgen Inc., Commack, NY, USA). The neuraminidase-treated samples were optimally diluted with PBST containing $1 \%$ bovine serum albumin (BSA), and a $50 \mu \mathrm{l} /$ well aliquot was added to the coated wells and incubated overnight at $4{ }^{\circ} \mathrm{C}$. After three washings with $250 \mu \mathrm{l}$ of PBST, a $100 \mu \mathrm{l}$ aliquot of horseradish peroxidase (HRP)-labeled goat anti-human IgA (1:5,000 dilution, IgG Fab fraction; MBL Co., LTD, Nagoya, Japan) was added to each well. Following a further $2 \mathrm{~h}$ incubation at $25^{\circ} \mathrm{C}$, the wells were washed 5 times with $250 \mu \mathrm{l}$ of PBST, and the signal was detected with $100 \mu$ l of $O$-phenylenediamine $(0.5 \mathrm{mg} / \mathrm{ml}$ Wako Pure Chemical Industries, LTD., Osaka, Japan) $-\mathrm{H}_{2} \mathrm{O}_{2}(0.1 \%)$ in $0.1 \mathrm{M}$ citrate phosphate buffer. The colour reaction was stopped with $100 \mu$ l of $1 \mathrm{M}$ sulfuric acid, and absorbance at $492 \mathrm{~nm}$ was measured.

Data analyses and statistics

To correct for variance between experimental conditions, results were expressed as standard deviation units (SDU), which were calculated as follows: SDU of each sample $=($ sample absorbance - mean absorbance of healthy controls)/standard deviation of healthy controls, where the mean and SD values of $\mathrm{HC}$ calculated in each experiment were used.

The statistics software package JMP10 (SAS Institute Inc., Cary, NC, USA) was used to analyze the data. The Kruskal-Wallis non-parametric test was used to identify significant differences in binding levels of the two monoclonal antibodies among IgAN, OKD, and HC groups (both antibodies $p<0.0001)$. The binding levels were then compared between one group and another in the three groups using the Steel-Dwass non-parametric test. Differences were regarded as statistically significant at a $p$ value $<0.05$.

\section{Results}

Comparisons of antibody reactivities among $\operatorname{IgAN}$, OKD and HC groups

The IgA binding levels of the two monoclonal antibodies in the IgAN group were similarly increased compared to levels in the OKD and $\mathrm{HC}$ groups. With the $35 \mathrm{~A} 12$ monoclonal antibody, significant differences were observed between IgAN vs. HC, and IgAN vs. OKD (both $p<0.0001$, Steel-Dwass non-parametric test, Fig. 2a);
Fig. 1 Dose response curves of $35 \mathrm{~A} 12$ (a) and $44 \mathrm{H} 8$ (b) monoclonal antibodies in four serum samples. Neuraminidase-treated human serum samples (three IgAN and one $\mathrm{HC}$ ) were serially diluted from $1 / 25$ to $1 / 6,400$
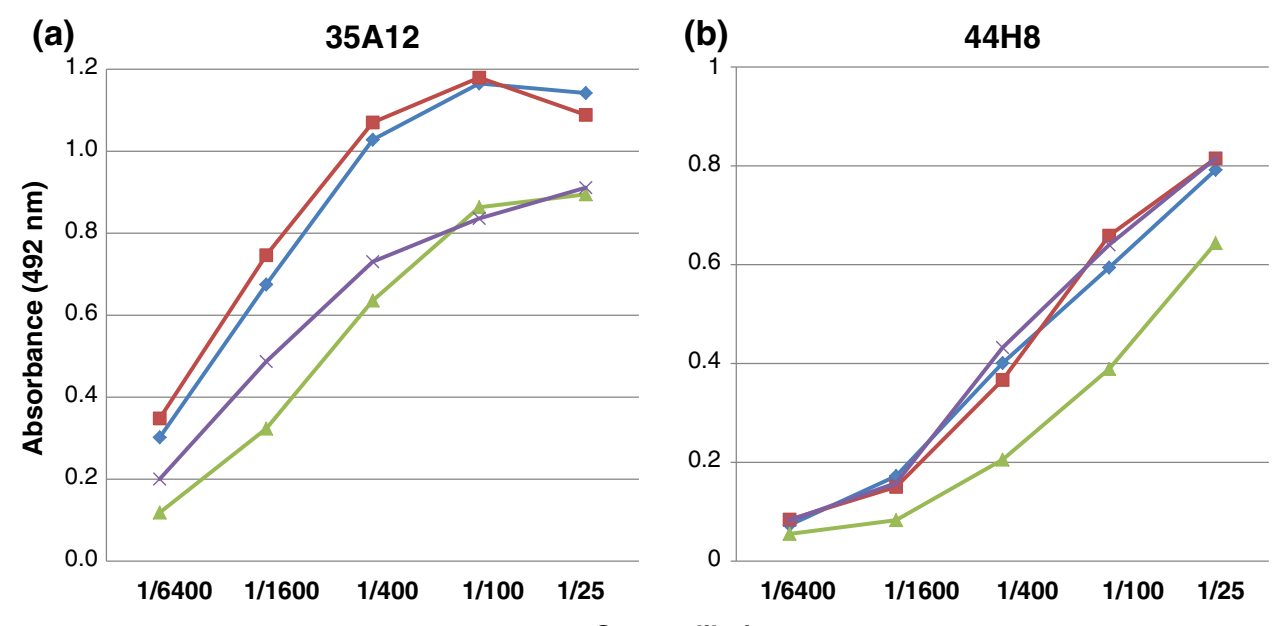

Serum dilution 
Fig. 2 Comparisons of $\operatorname{IgA}$ levels bound to two monoclonal antibodies in the HC, IgAN and OKD groups (a 35A12,

b 44H8). a The IgA binding level of 35A12 monoclonal antibody in the IgAN group was significantly increased compared to the OKD and HC groups (both $p<0.0001$ ). There was no significant difference between $\mathrm{HC}$ and $\mathrm{OKD}$ ( $p=0.113$ ). b The IgA binding level of 44H8 antibody in the IgAN groups was significantly increased compared to the $\mathrm{HC}$ and OKD groups (both $p<0.0001)$. There was no significant difference between $\mathrm{HC}$ and $\mathrm{OKD}(p=0.831)$
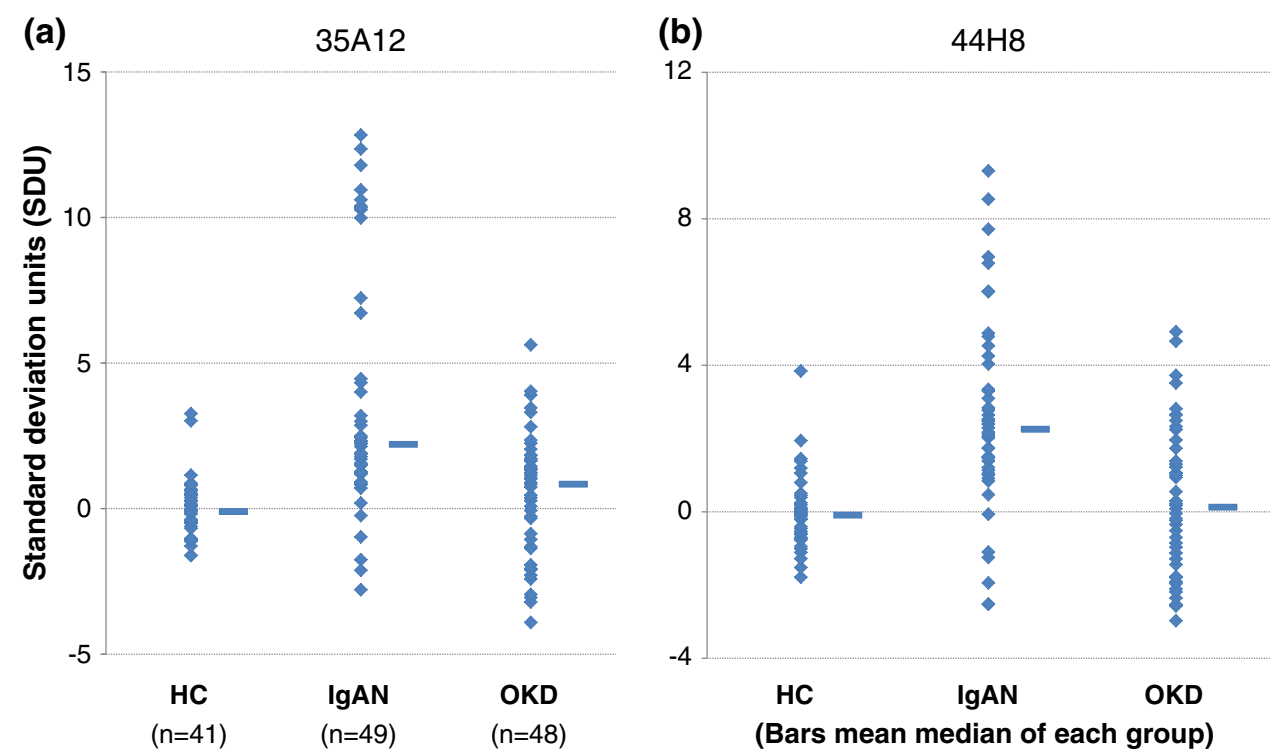

however, no difference was found between $\mathrm{HC}$ vs. OKD $(p=0.113)$. With the $44 \mathrm{H} 8$ antibody, a significant increase was observed in the $\operatorname{IgAN}$ group compared to the $\mathrm{HC}$ and OKD groups (both $p<0.0001$, Fig. 2b). There was no significant difference between $\mathrm{HC}$ and $\mathrm{OKD}$ $(p=0.831)$.

Correlation between B35A12 and 44H8 antibodies

The IgA binding levels of 35A12 and 44H8 antibodies were strongly correlated in individual samples $\left(R^{2}=0.737\right)$ (Fig. 3).

Receiver operating characteristic (ROC) curves

The areas under the curve (AUC) calculated from the ROC curves for the IgAN group are summarized in Table 2. The AUC values of $\operatorname{IgAN}$ patients for $35 \mathrm{~A} 12$ against $\mathrm{HC}$ (Fig. 4a) and OKD (Fig. 4b) were 0.861 and 0.741, respectively. The AUC values for $44 \mathrm{H} 8$ were 0.854 (Fig. 5a) and 0.760 (Fig. 5b) for $\mathrm{HC}$ and OKD, respectively.

\section{Discussion}

Human $\operatorname{IgA} 1$ has a unique hinge structure due to the presence of mucin-like $O$-linked oligosaccharides [16]. The core peptide consists of a proline-, serine-, and threoninerich amino acid sequence in which the serine and threonine residues are $O$-glycosylated with structures consisting of neuraminic acid, galactose and $N$-acetylgalactosamine, with microheterogeneity observed. In the past two decades, it has been demonstrated that certain abnormalities exist in

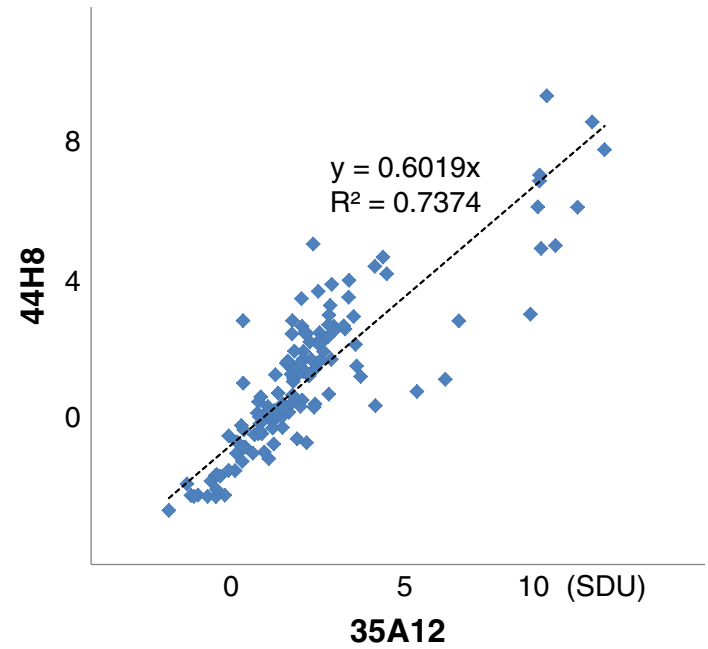

Fig. 3 Correlation between 35A12 and 44H8 IgA binding levels. There was a strong correlation between the results obtained for the $35 \mathrm{~A} 12$ and $44 \mathrm{H} 8$ antibodies $\left(R^{2}=0.737\right)$, suggesting that the two monoclonal antibodies recognize a similar epitope of sHGP, and that the results of the IgA binding levels are reproducible

Table 2 Summary of AUC of ROC curves for each monoclonal antibody

\begin{tabular}{lll}
\hline $\begin{array}{l}\text { Monoclonal } \\
\text { antibody }\end{array}$ & $\begin{array}{l}\text { vs. Healthy } \\
\text { controls }\end{array}$ & $\begin{array}{l}\text { vs. Other kidney } \\
\text { diseases }\end{array}$ \\
\hline $35 \mathrm{~A} 12$ & 0.861 & 0.741 \\
$44 \mathrm{H} 8$ & 0.854 & 0.760 \\
\hline
\end{tabular}

the $O$-glycan structures of the $\operatorname{IgA} 1$ hinge region in $\operatorname{IgAN}$. Although these abnormalities have been suggested to be due to hypo-glycosylation (especially hypo-galactosylation), the precise structure of these glycans is not yet clear. The HAA-assay was capable of demonstrating a significant 
Fig. 4 Receiver operating characteristic (ROC) curves of the 35A12 antibody between the IgAN and $\mathrm{HC}$ groups, and the IgAN and OKD groups. The AUC values of IgAN patients for 35A12 against $\mathrm{HC}$ (a) and OKD (b) were 0.861 and 0.741 , respectively

Fig. 5 Receiver operating characteristic (ROC) curves of the 44H8 antibody between the IgAN and $\mathrm{HC}$ groups, and the IgAN and OKD groups. The AUC values of IgAN patients for $44 \mathrm{H} 8$ against $\mathrm{HC}$ (a) and OKD (b) were 0.854 and 0.760 , respectively (a)

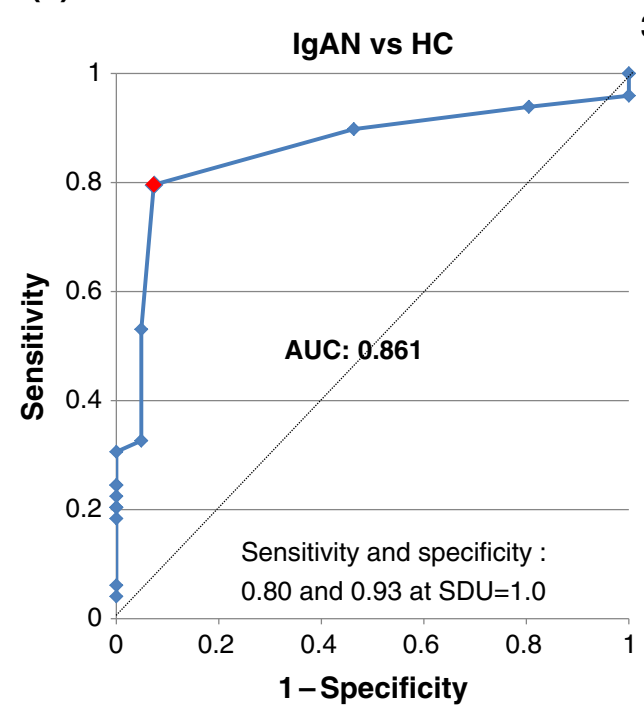

(a)

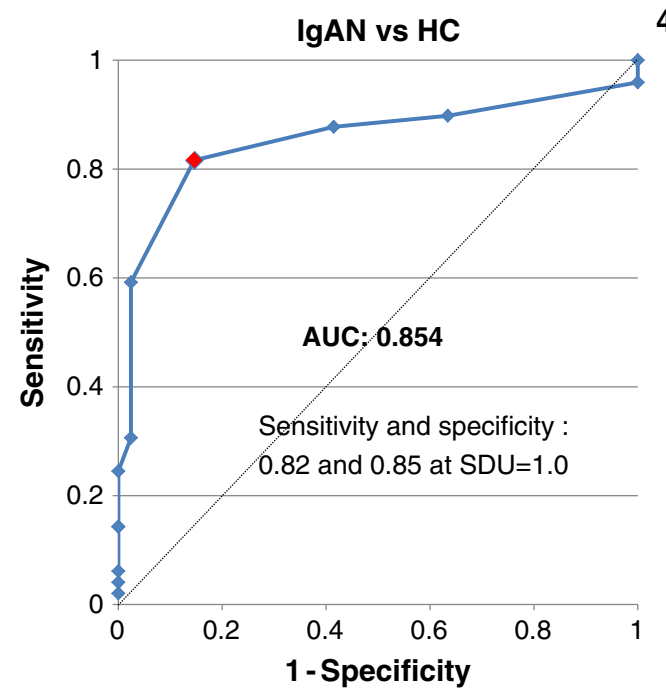

(b)

35A12

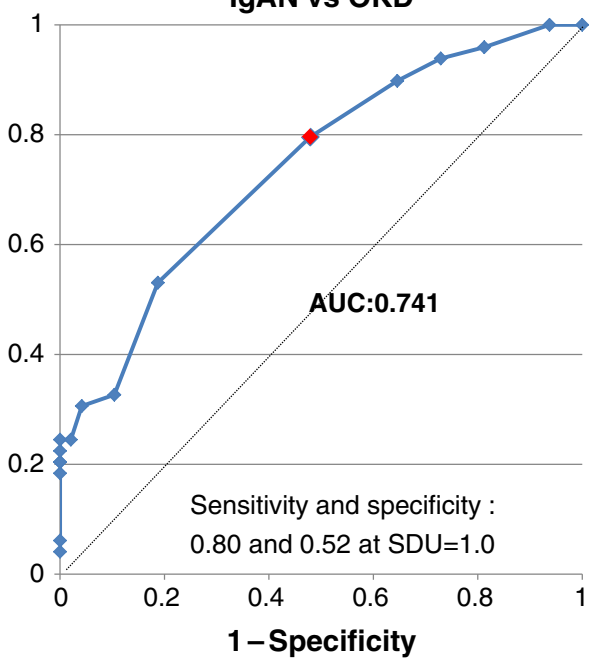

(b)

$44 \mathrm{H} 8$

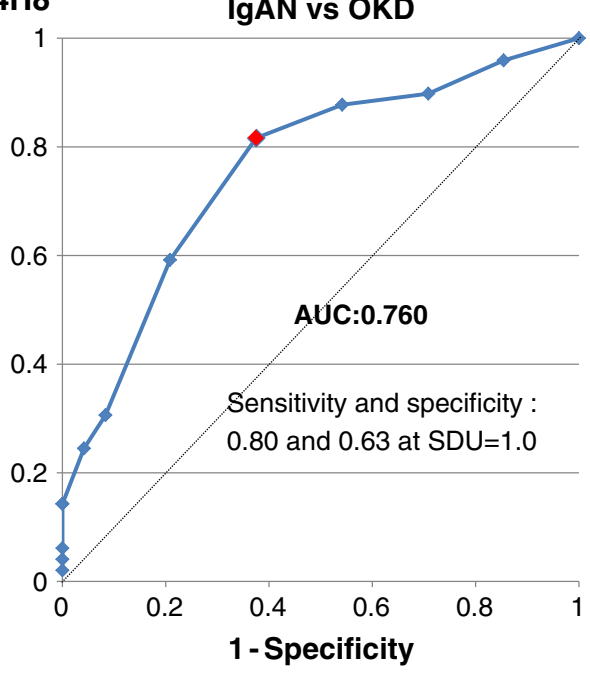

increase in exposed GalNAc residues in IgAN samples. In other words, IgAN patients had fewer Gal residues in the IgA1 hinge region. However, the relatively weak binding affinity of lectins to the corresponding carbohydrates made the assay unstable and insufficiently reproducible.

Previously, we developed a polyclonal antibody using a synthetic hinge glycopeptide [15]. The polyclonal antibody bound more strongly to asialo/agalacto-IgA1 than untreated and asialo-IgA1, suggesting that the antibody recognized hinge peptides containing GalNAc residues. Further, the binding levels of the polyclonal antibody were closely related to those observed using the HAA ELISA in the same patient sera $\left(R^{2}=0.5964\right)$, with IgAN binding significantly increased compared to the OKD and HC groups. However, the polyclonal antibody was far inferior to the monoclonal antibodies introduced in this study.
In this study, we developed two monoclonal antibodies against a synthetic hinge glycopeptide, which is regarded as a model of hypo-galactosylated IgA1 hinge protein. The increased antigenicity against the antibody in IgAN suggested that IgAN patients have hypo-glycosylated IgA1 with exposed GalNAc residues on the hinge core peptide.

It is unlikely that the two monoclonal antibodies are currently clinically applicable as substitutes for renal biopsy. Both monoclonal antibodies exhibited insufficient specificity for differentiating IgAN from other kidney diseases. However, as the sensitivities were acceptable for clinical applications, it may be possible to utilize them as non-invasive tools for IgAN screening. It is expected that the development of a highly specific antibody could replace the invasive renal biopsy method for diagnosing IgAN. 
Acknowledgments The authors thank Dr. T. Tomiyama for producing the monoclonal antibodies. We also thank Ms. M. Sakata, Ms. A. Kamiya and Mr. H. Itoh for their superb technical assistance and data analyses. This work was supported by grants from Fujita Health University Research Foundation and by New Energy and Industrial Technology Organization (NEDO) supported by Japanese Government. The monoclonal antibody is the subject of a patent application (Domestic patent reception No.: 51302302502).

Conflict of interest $\mathrm{YH}$ received a research support grant from Sumitomo Dainippon Pharma Co., Ltd.. YY received research funds from Otsuka Pharmaceutical Co., Ltd., Kyowa Hakko Kirin Co., Ltd. and Baxter Ltd.. No other conflict of interest to declare.

Open Access This article is distributed under the terms of the Creative Commons Attribution License which permits any use, distribution, and reproduction in any medium, provided the original author(s) and the source are credited.

\section{References}

1. Czerkinsky C, Koopman WJ, Jackson S et al (1986) Circulating immune complexes and immunoglobulin A rheumatoid factor in patients with mesangial immunoglobulin A nephropathies. J Clin Invest. 77:1931-1938

2. Hiki Y, Saitoh M, Kobayashi Y (1991) Serum IgA class anti-IgA antibody in IgA nephropathy. Nephron 59:552-560

3. Kokubo T, Hashizume K, Iwase H et al (2000) Humoral immunity against the proline-rich peptide epitope of the IgA1 hinge region in IgA nephropathy. Nephrol Dial Transplant 15:28-33

4. Nakamura I, Iwase H, Arai K et al (2004) Detection of gender difference and epitope specificity of IgG antibody activity against IgA1 hinge portion in IgA nephropathy patients by using synthetic hinge peptide and glycopeptide probes. Nephrology 9:26-30

5. Iwase H, Ishii-Karakasa I, Fujii E et al (1992) Analysis of glycoform of O-glycan from human myeloma immunoglobulin A1 by gas-phase hydrazinolysis following pyridylamination of oligosaccharides. Anal Biochem 206:202-205

6. Allen AC, Harper SJ, Feehally J (1995) Galactosylation of N- and O-linked carbohydrate moieties of IgA1 and IgG in IgA nephropathy. Clin Exp Immunol 100:470-477

7. Hiki Y, Iwase H, Kokubo T et al (1996) Association of asialogalactosyl beta 1-3 $N$-acetylgalactosamine on the hinge with a conformational instability of Jacalin-reactive immunoglobulin A1 in immunoglobulin A nephropathy. J Am Soc Nephrol 7:955-960

8. Kokubo T, Hiki Y, Iwase H et al (1998) Protective role of IgA1 glycans against IgA1 self-aggregation and adhesion to extracellular matrix proteins. J Am Soc Nephrol 9:2048-2054

9. Hiki Y, Kokubo T, Iwase H et al (1999) Underglycosylation of IgA1 hinge plays a certain role for its glomerular deposition in IgA nephropathy. J Am Soc Nephrol 10:760-769

10. Tomana M, Novak J, Julian BA et al (1999) Circulating immune complexes in IgA nephropathy consist of IgA1 with galactosedeficient hinge region and antiglycan antibodies. J Clin Invest 104:73-81

11. Hiki Y, Odani H, Takahashi M et al (2001) Mass spectrometry proves under $O$-glycosylation of glomerular $\operatorname{IgA} 1$ in $\operatorname{IgA}$ nephropathy. Kidney Int 59:1077-1085

12. Allen AC, Bailey EM, Brenchley PE et al (2001) Mesangial IgA1 in IgA nephropathy exhibits aberrant $O$-glycosylation: observations in three patients. Kidney Int 60:969-973

13. Moldoveanu Z, Wyatt RJ, Lee JY et al (2007) Patients with IgA nephropathy have increased serum galactose-deficient IgA1 levels. Kidney Int 71:1148-1154

14. Shimozato S, Hiki Y, Odani H et al (2008) Serum under-galactosylated IgA1 is increased in Japanese patients with $\operatorname{IgA}$ nephropathy. Nephrol Dial Transplant 23:1931-1939

15. Yamamoto K, Hori H, Yamamoto Y et al (2012) Development of a polyclonal antibody against synthetic human immunoglobulin A1 hinge glycopeptide. J Glycobiology 1:2-4

16. Mattu TS, Pleass RJ, Willis AC et al (1998) The glycosylation and structure of human serum $\operatorname{IgA} 1, \mathrm{Fab}$ and $\mathrm{Fc}$ regions and the role of $\mathrm{N}$-glycosylation on $\mathrm{Fc}$ alpha receptor interactions. J Biol Chem 273:2260-2272 